\title{
Nouvelles acquisitions patrimoniales
}

\section{Daniel Bornemann}

\section{(2) OpenEdition}

Journals

Édition électronique

URL : http://journals.openedition.org/rbnu/1045

DOI : $10.4000 /$ rbnu. 1045

ISSN : 2679-6104

\section{Éditeur}

Bibliothèque nationale et universitaire de Strasbourg

\section{Édition imprimée}

Date de publication : 1 mai 2017

Pagination : 122-125

ISBN : 9782859230661

ISSN : 2109-2761

\section{Référence électronique}

Daniel Bornemann, « Nouvelles acquisitions patrimoniales », La Revue de la BNU [En ligne], 15 | 2017, mis en ligne le 01 novembre 2019, consulté le 13 décembre 2020. URL : http:// journals.openedition.org/rbnu/1045; DOI : https://doi.org/10.4000/rbnu.1045

\section{(C) $10(0$}

La Revue de la BNU est mise à disposition selon les termes de la Licence Creative Commons Attribution - Pas d'Utilisation Commerciale - Partage dans les Mêmes Conditions 4.0 International. 


\section{Nouvelles acquisitions patrimoniales $\downarrow$ \\ PAR DANIEL BORNEMANN}

\section{Un diurnal dominicain}

Acquérir un manuscrit médiéval est pour une bibliothèque universitaire un fait exceptionnel. La BNU réussit pourtant assez régulièrement à faire entrer dans ses fonds des " codices " qui témoignent de la culture et de l'art du livre dans notre région au Moyen Âge. Ce diurnal dominicain du $14^{\mathrm{e}}$ siècle vient s'ajouter à notre collection riche d'environ 700 manuscrits médiévaux, de toutes origines. Celui-ci semble bien ancré dans le patrimoine local, comme le montrent quelques éléments qui permettent de l'attribuer à notre cité : ainsi la mention d'un « Dominus Dietericus de Burchein » dans le calendrier, nom que l'on peut rattacher à Strasbourg, et la présence de sainte Odile parmi les saints dans une addition au codex d'origine (au f. 319 verso).

Comme son nom l'indique, un diurnal est un manuscrit qui contient les heures de l'office divin pour la journée. Comme le bréviaire, dont il est très proche par le contenu et par la forme, il est généralement portatif. Il ne contient pas, à la différence du bréviaire, la liturgie des matines. L'ouvrage s'ouvre sur un calendrier, puis propose une introduction générale au diurnal. Le temporal y fait suite, contenant les offices des jours ainsi que l'office pour la dédicace d'une église. Puis vient le sanctoral, pour les offices des jours, avec notamment les offices pour Pierre Martyr ou pour la translation de Dominique. Le commun des saints pour les offices des jours est suivi d'hymnes et de prières. Enfin, on y trouve l'office des Heures de la Vierge et le psautier. Des additions ultérieures au $14^{\mathrm{e}}$ siècle sont venues enrichir cet ensemble - on peut les dater des $15^{\mathrm{e}}$ et $16^{\mathrm{e}}$ siècles. On y a notamment ajouté des liturgies concernant les matines, ce qui rapproche le manuscrit d'un bréviaire. La reliure actuelle du volume semble être du $16^{\mathrm{e}}$ siècle au plus tôt, et a nécessité en des temps lointains un court rognage des marges de tête et de gouttière. Ses propriétaires successifs n'y ont pas laissé de marques d'appartenance, de sorte que l'on ignore sa trajectoire dans le temps.

Notre diurnal est richement décoré. Sans contenir aucune enluminure figurative, ni aucune application de métaux précieux, il compte un très grand nombre (jusqu'à plusieurs par page) de lettrines, chacune pourvue de proliférations marginales, et une page plus particulièrement décorée. L'écriture en noir et en rouge peut être qualifiée de lettre de forme proche de la «textura ", avec cependant des arrondis.

On rencontre dans les collections des bibliothèques d'Alsace et $\mathrm{du}$ Bade (et même des États-Unis, par exemple au Boston Museum of fine arts) des manuscrits contenant des pages décorées du même style, avec des éléments graphiques très proches. Le cadre cantonné de quatre médaillons circulaires habités chacun par un petit dragon bipède ou par des fleurons, se retrouve dans plusieurs manuscrits qui donnent véritablement l'impression d'être l'œuvre d'une même main. La grande lettrine E (de « Ecce dies... »), haute de cinq lignes, est également habitée par des créatures fantastiques (dragons bipèdes) s'affrontant ou se poursuivant. Les marges contiennent un grand nombre de petits personnages, animaux ou humains, esquissés d'un trait de plume très délié. Par son décor, l'ouvrage s'apparente notamment au Grand mémorial de la Commanderie de Saint-Jean de l'Île-Verte, de Strasbourg, conservé à la BNU, et au Psautier de Colmar (conservé à la Bibliothèque municipale de cette ville).

Ce diurnal dominicain vient donc s'ajouter à une série de manuscrits qui présentent un type de décor très proche, voire identique, et sont sans doute issus d'un même atelier. C'est donc une acquisition dont l'étude par les spécialistes pourra apporter des pièces supplémentaires au dossier de l'enluminure strasbourgeoise du Moyen Âge.

COTE DU MANUSCRIT : MS.7.122 337 (+ 2) f., 95 X 65 mm 


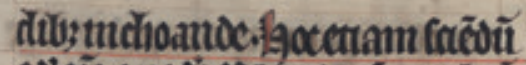

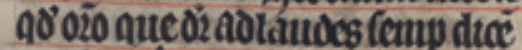
oa cet dotitam fextam etnonam

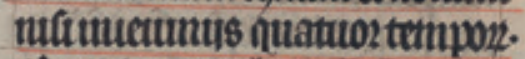
aductutus ctinditobus dicos togati onti detuturectadis qun aqutut x coxen cratuomlautoustufip fias mâl.etinditobsdicls mgationit.

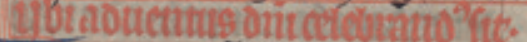
E Ducutus oin femp coledmature etcilet celebtatimionmenta nue

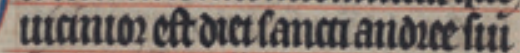

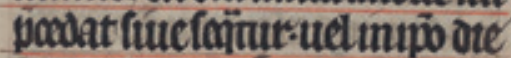
fantuanotrenumetiomintcace tecitt. ommianueadanoes et ad hozas dicu protume adoucutuî

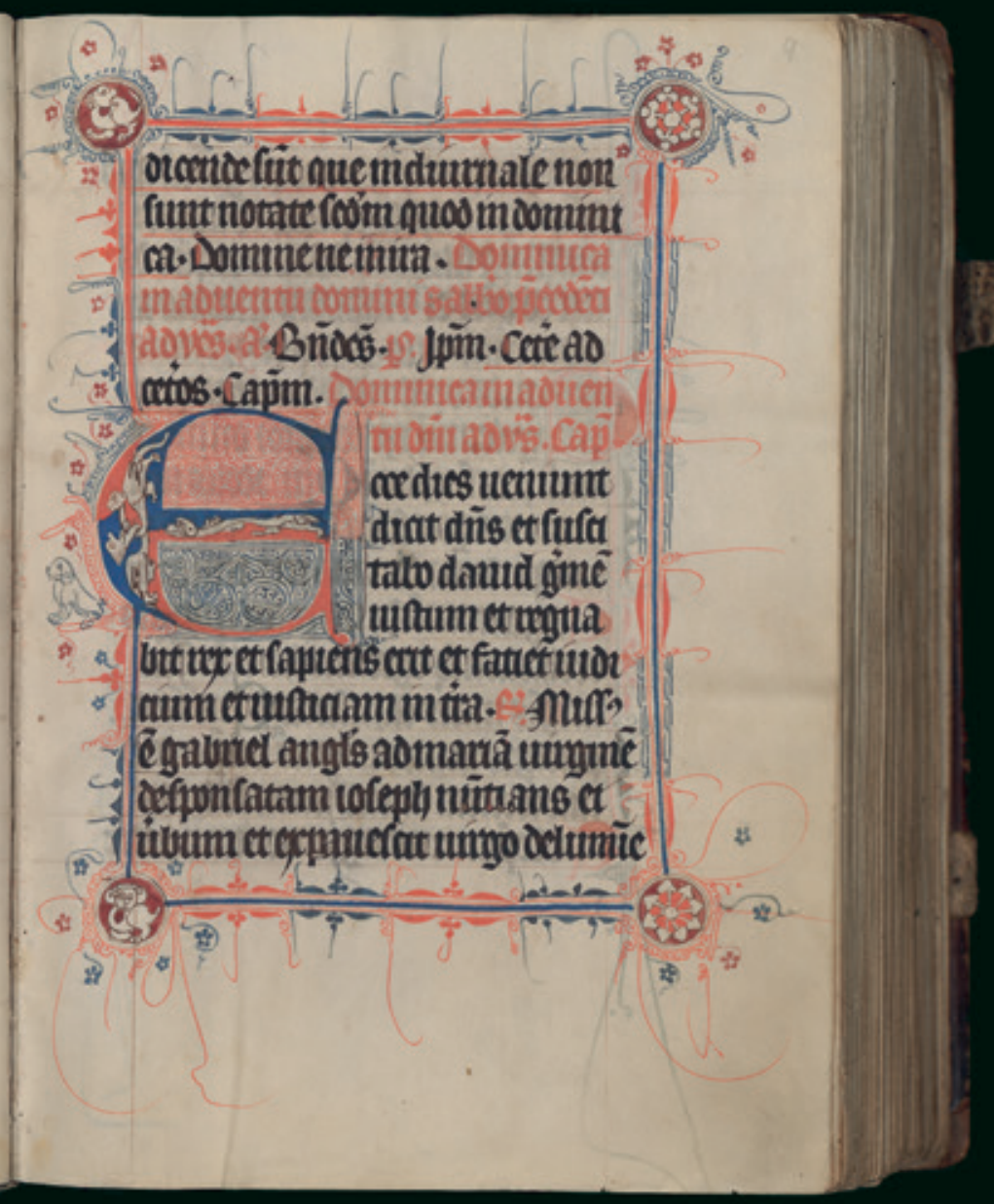




\section{Nouvelles acquisitions patrimoniales \\ $\downarrow$ \\ PAR DANIEL BORNEMANN}

Guernica... ein Fanal

des Faschismus (Strasbourg, Prométhée, 1937). Livret édité par l'émigration allemande antifasciste à Strasbourg.

Il y a quatre-vingt ans, le 26 avril 1937, un déluge de feu s'abattait sur la petite ville basque de Guernica. Prélude à d'autres bombardements qui allaient suivre durant les années de la Seconde Guerre mondiale, ce nom que l'histoire et l'art ont rendu célèbre résonne à présent dans les consciences européennes. Au cœur de la guerre civile espagnole, cet événement posa aux protagonistes divers problèmes. Qui était le responsable de cette destruction aveugle ? Le parti franquiste, les « rouges » ou d'autres encore : les soldats allemands ? La vérité sur l'opération de la légion Condor dirigée par le général von Richthofen ne fut pas avouée avant de nombreuses années. Franco voulut se disculper en accusant les Allemands, puis les « rouges ", de ce massacre. Mais les réseaux de soutien à la cause des républicains espagnols ne se laissèrent pas si facilement tromper. Ce modeste livret illustré de 16 pages en est une trace significative. C'est une maison d'édition basée à Strasbourg qui l'édita dès 1937 pour désigner clairement l'acteur principal de l'attaque : le régime hitlérien et son bras armé.

L'auteur de la plaquette, Franz Lang, dont le véritable nom est Jakob Rosner, est un journaliste autrichien, né en Galicie en 1890,

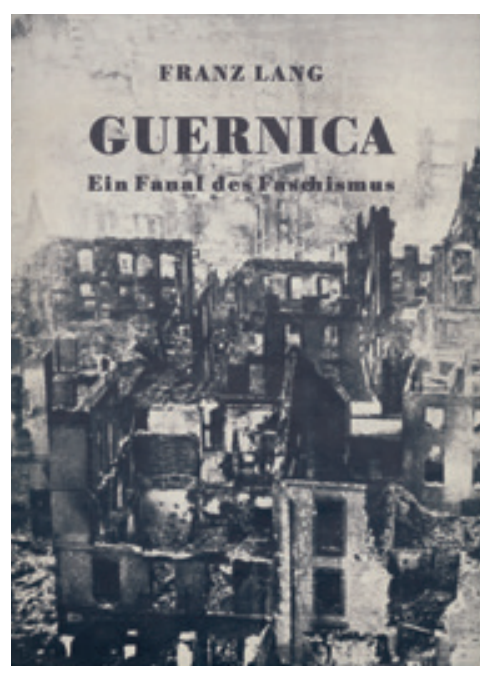

mort à Vienne en 1970, qui a fait une carrière de publiciste et d'homme politique marxiste, fut membre $\mathrm{du}$ parti communiste autrichien et du Komintern, et collabora avec Dimitrov dans sa période berlinoise. Il traversa ensuite la guerre en exil, entre Suède et Russie.

Ce livret est aujourd'hui d'une grande rareté, car il a échappé au dépôt légal français. La BnF ne le possède pas (en tout cas d'après ses catalogues) et le seul exemplaire français semble être celui que la BNU a pu acquérir. Le dépôt légal imprimeur n'a pas non plus joué, puisque l'ouvrage a été imprimé en Suisse. Notons d'ailleurs que la même plaquette a été éditée la même année par un éditeur zurichois appelé Freie Schweiz (Suisse libre).

Ce document ainsi qu'un titre apparenté, que la BNU a aussi acheté et dont le titre est Guadalajara:
Eine Niederlage des Faschismus. Mit Beiträgen von Michail Kolzow, Ilja Ehrenburg, spanische Milizoffizieren und anderen, également de 1937, ont donc échappé aux collections des bibliothèques françaises, et même à la collection des " alsatiques » de la BNU. En Allemagne, seules deux bibliothèques semblent posséder ces livrets.

Rappelons que le personnage de Prométhée (qui a donné son nom à la maison d'édition strasbourgeoise), d'après la tragédie d'Eschyle, est puni pour avoir donné à l'humanité le feu et une série d'autres bienfaits, contrevenant à la volonté de Zeus. Grâce à lui, les hommes ont acquis à la fois la pensée et la conscience. Ce nom a été choisi pour cette structure d'édition, d'abord sise à Strasbourg, au moins à partir de 1935, puis à partir de 1938 à Paris (d'après ce qu'on peut déduire des publications). Cette littérature de l'exil, du combat, est située dans les marges des circuits officiels de l'édition. Ces livrets sont donc les fragiles témoignages d'une écriture de lutte, de tactiques de diffusion et d'édition particulières. Le catalogue collectif des bibliothèques universitaires (le SUDOC) permet de repérer bien d'autres publications de cette maison d'édition, durant les années 1930, imprimées en France (Strasbourg puis Paris) et en langue allemande, et portant sur le marxisme et la lutte contre l'hitlérisme.

COTE : R.250.263

$16 \mathrm{p}$. 


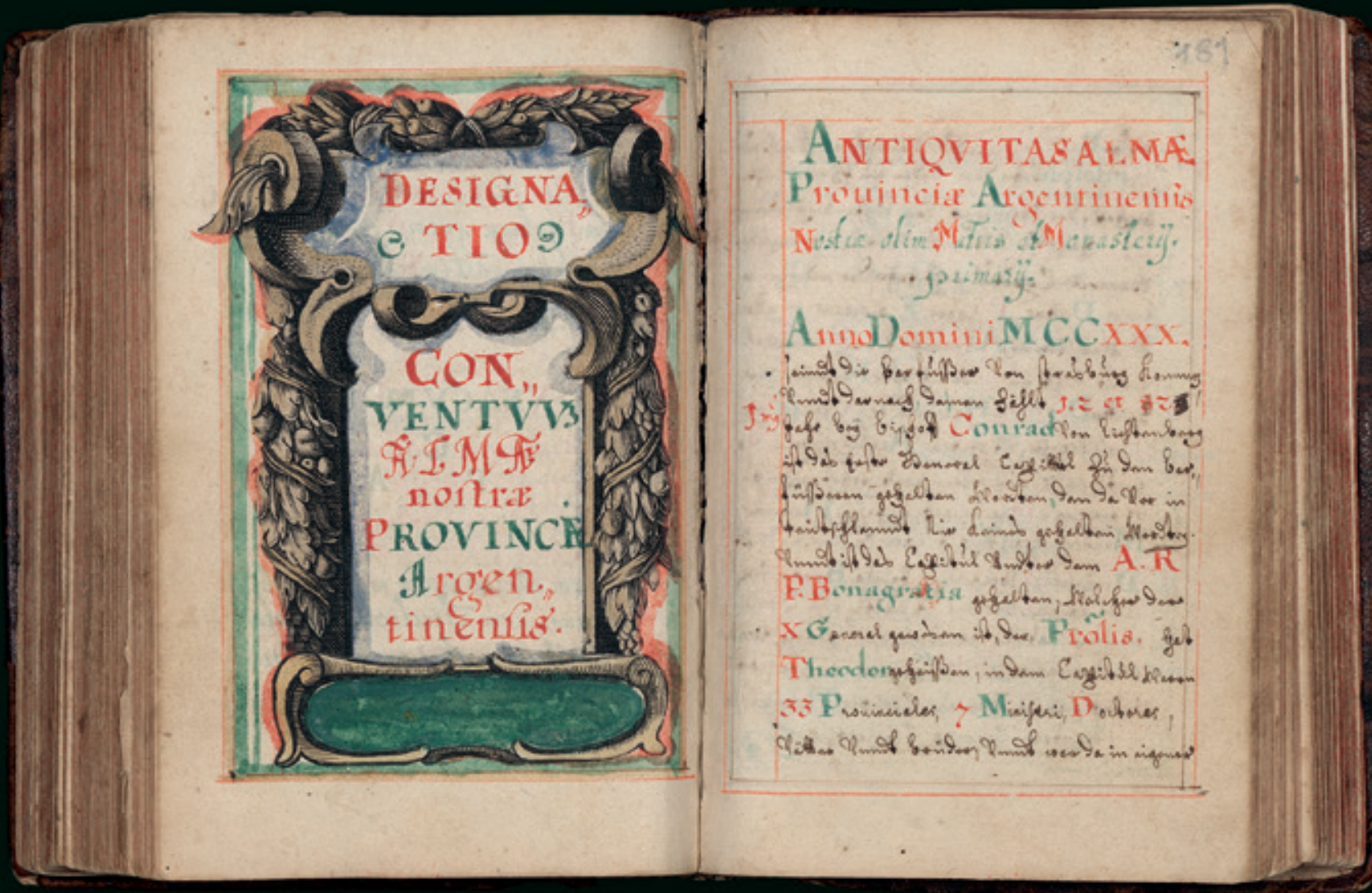

Regula et Vita fratrum

minorum, petit manuscrit de Chrysostome Drölling

Ce petit manuscrit, dont le format rappelle les règles franciscaines imprimées qui sont généralement de très petits volumes au format in-12, est le témoignage de la persistance du manuscrit de copie bien au-delà de l'invention de l'imprimerie. Nous sommes ici en 1732 et le franciscain Chrysostome Drölling écrit sa propre règle franciscaine latine et en fait un très beau manuscrit décoré et calligraphié avec soin. Il serait plus exact de dire que le copiste conçoit son manuscrit en utilisant pour les pages de titre intermédiaires des éléments gravés sur cuivre, qu'il adapte à son ouvrage. Les pages de titre gravées, insérées dans le manuscrit, sont ensuite coloriées avec soin et nuances, pourvues d'encadrements colorés qui les intègrent au reste du manuscrit, et enfin remplies par le titrage calligraphié en couleurs, alternant les écritures romaines capitales et minuscules, et même gothique et cursive.

Cette règle contient les parties suivantes : Regula et Vita fratrum minorum (f. 1-35) ; Enchiridion fratrum minorum complectens regulae nostrae, Expositionem ad usum fratrum laicorum (f. 36-71) ; Officium ceremoniarii (f. 72-131) ; Conditiones sacrum ordinem nostrum (f. 132-146) ; Modus seu brevis instructio ad meditationes (f. 147-173) ; Comendationes pro anima defuncti fratris (f. 174180) ; Designatio conventuus alma nostrae provinciae argentinensis (f. 181-189r) ; Variae benedictiones indumentorum sacerdotalia $(189 \mathrm{v}-$ 198) ; Benedictio imaginis beatae Mariae virginis (f. 199-202) ; Novae crucis erigendae benedictio (f. 203220) ; Statuta provincalia (f. 221233) ; Duodecim vitae religiosae monita ad veram perfectionem tendentia singulis diebus recolligenda (f. 234-282).

Sa reliure est plein veau brun à 1 filet à froid, le dos à 4 nerfs est décoré de 5 fleurons poussés à froid.

COTE : MS.7.123

$285 \mathrm{X} 129 \mathrm{~mm}, 282 \mathrm{f}$. 